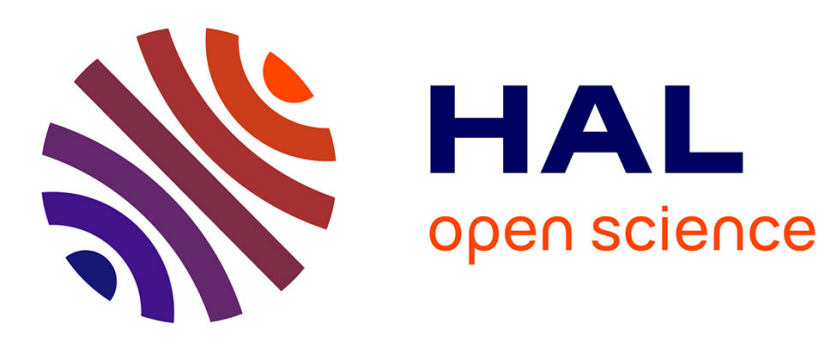

\title{
Implantable cardiac defibrillator leads dysfunction after LVAD implantation
}

Vincent Galand, Christophe Leclercq, Hamed Bourenane, Stéphane Boulé, André Vincentelli, Philippe Maury, Pierre Mondoly, François Picard, Nicolas Welte, Michel Kindo, et al.

\section{To cite this version:}

Vincent Galand, Christophe Leclercq, Hamed Bourenane, Stéphane Boulé, André Vincentelli, et al.. Implantable cardiac defibrillator leads dysfunction after LVAD implantation. PACE - Pacing and Clinical Electrophysiology, 2020, 43 (11), pp.1309-1317. 10.1111/pace.14004 . hal-02890820

\section{HAL Id: hal-02890820 \\ https://hal.science/hal-02890820}

Submitted on $30 \mathrm{Jul} 2020$

HAL is a multi-disciplinary open access archive for the deposit and dissemination of scientific research documents, whether they are published or not. The documents may come from teaching and research institutions in France or abroad, or from public or private research centers.
L'archive ouverte pluridisciplinaire HAL, est destinée au dépôt et à la diffusion de documents scientifiques de niveau recherche, publiés ou non, émanant des établissements d'enseignement et de recherche français ou étrangers, des laboratoires publics ou privés. 


\section{Implantable cardiac defibrillator leads dysfunction after LVAD implantation}

Vincent Galand MD ${ }^{1} \quad$ C Christophe Leclercq MD, PhD ${ }^{1} \quad$ | Hamed Bourenane MD ${ }^{1}$ |

Stéphane Boulé $\mathrm{MD}^{2}$ | André Vincentelli MD, $\mathrm{PhD}^{2}$ | Philippe Maury MD, $\mathrm{PhD}^{3}$

Pierre Mondoly MD ${ }^{3}$ | François Picard MD ${ }^{4}$ | Nicolas Welté MD ${ }^{4}$ | Michel Kindo MD, PhD $^{5}$ | Thomas Cardi MD ${ }^{5}$ | Jean-Luc Pasquié MD ${ }^{6}$ | Philippe Gaudard MD | Jean-Baptiste Gourraud MD, $\mathrm{PhD}^{8}$ ｜ Vincent Probst MD, $\mathrm{PhD}^{8} \quad$ | Pascal Defaye $\mathrm{MD}^{9}$ । Aude Boignard MD ${ }^{9}$ | Marylou Para MD ${ }^{10}$ | Vincent Algalarrondo MD ${ }^{10}$ |

Edeline Pelcé MD ${ }^{11}$ | Vlad Gariboldi MD, PhD ${ }^{11}$ | Matteo Pozzi MD, PhD ${ }^{12}$ | Jean-François Obadia MDPhD ${ }^{12} \quad$ Frédéric Anselme MD ${ }^{13} \quad$ Pierre-Yves Litzler MD, PhD $^{13}$ | Katrien Blanchart MD ${ }^{14}$ | Gerard Babatasi MD, PhD ${ }^{14}$ | Fabien Garnier MD ${ }^{15}$ Marie Bielefeld MD ${ }^{15}$ | David Hamon MD, PhD ${ }^{16}$ | Nicolas Lellouche MD, $\mathrm{PhD}^{16}$ | Thierry Bourguignon MD ${ }^{17} \quad$ Bertrand Pierre MD ${ }^{17}$ | Romain Eschalier MD, PhD ${ }^{18}$ Nicolas D'Ostrevy MD ${ }^{18}$ | Emilie Varlet MD ${ }^{19}$ | Eloi Marijon MD, PhD ${ }^{19}$ | Hugues Blangy MD ${ }^{20}$ | Nicolas Sadoul MD, PhD ${ }^{20}$ | Erwan Flécher MD, PhD ${ }^{1}$ | Raphaël P. Martins MD, PhD ${ }^{1}$

${ }^{1}$ CHU Rennes, INSERM, University of Rennes, Rennes, France

${ }^{2}$ Cardiac Intensive Care Unit, Department of Cardiology and Cardiac Surgery, CHU Lille, Institut Coeur-Poumons, Lille, France

${ }^{3}$ Centre Hospitalier Universitaire de Toulouse, Toulouse, France

${ }^{4}$ Hôpital Cardiologique du Haut-Lévêque, LIRYC institute, Université Bordeaux, Bordeaux, France

${ }^{5}$ Département de chirurgie cardiovasculaire, hôpitaux universitaires de Strasbourg, Strasbourg, France

${ }^{6}$ Department of Cardiology, CHU Montpellier, Montpellier, France

${ }^{7}$ Department of Anesthesiology and Critical Care Medicine, PhyMedExp, University of Montpellier, INSERM, CNRS, CHU Montpellier, Montpellier, France

${ }^{8}$ Department of Cardiology and Heart Transplantation Unit, CHU Nantes, Nantes, France

9 Department of Cardiology and Cardiovascular Surgery, CHU Michallon, Grenoble, France

${ }^{10}$ Department of Cardiology and Cardiac Surgery, Bichat-Hospital, Paris, France

${ }^{11}$ Department of Cardiac Surgery, La Timone Hospital, Marseille, France

12 Department of Cardiac Surgery, “Louis Pradel” Cardiologic Hospital, Lyon, France

${ }^{13}$ Department of Cardiology and Cardiovascular Surgery, Hospital Charles Nicolle, Rouen, France

${ }^{14}$ Department of Cardiology and Cardiac Surgery, University of Caen and University Hospital of Caen, Caen, France

${ }^{15}$ Department of Cardiology and Cardiac Surgery, University Hospital, Dijon, France

${ }^{16}$ Department of Cardiology and Cardiac Surgery, AP-HP CHU Henri Mondor, Créteil, France

${ }^{17}$ Department of Cardiology and Cardiac Surgery, Tours University Hospital, Tours, France

${ }^{18}$ Cardiology Department, CHU Clermont-Ferrand, Clermont-Ferrand, France

${ }^{19}$ Cardiology Department, European Georges Pompidou Hospital, Paris, France

20 Department of Cardiology and Cardiac Surgery, CHU de Nancy, Hopital de Brabois, Nancy, France 


\section{Correspondence}

Vincent Galand, MD, Service de Cardiologie et Maladies Vasculaires, $\mathrm{CHU}$ de Rennes, 2 rue Henri Le Guilloux, 35000 Rennes, France.

Email:vincent.galand35@gmail.com

Funding information Fédération Française de Cardiologie, Grant/Award Number: NA

\section{Abstract}

Background: Implantable cardioverter-defibrillator (ICD) lead dysfunction has been reported after left ventricular assist device (LVAD) implantation in limited singlecenter studies. We aimed at describing and characterizing the incidence of ICD lead parameters dysfunction after LVAD implantation.

Methods: Among the 652 patients enrolled in the ASSIST-ICD study, only patients with an ICD prior to LVAD were included ( $n=401$ ). ICD lead parameters dysfunction following LVAD implantation is defined as follows: (a) $>50 \%$ decrease in sensing threshold, (b) pacing lead impedance increase/decrease by $>100 \Omega$, and $(c)>50 \%$ increase in pacing threshold.

Results: One hundred twenty-two patients with an ICD prior to LVAD had available ICD interrogation reports prior and after LVAD. A total of 67 (55\%) patients exhibited at least one significant lead dysfunction: 17 (15\%) exhibited $>50 \%$ decrease in right ventricular (RV) sensing, 51 (42\%) had $>100 \Omega$ increase/decrease in RV pacing impedance, and 24 (20\%) experienced $>50 \%$ increase in RV pacing threshold. A total of 52 patients experienced ventricular arrhythmia during follow-up and all were successfully detected and treated by the device. All lead dysfunction could be managed conservatively.

Conclusion: More than $50 \%$ of LVAD-recipients may experience $>1$ significant change in lead parameters but none had severe clinical consequences.

\section{KEYWORDS}

implantable cardioverter defibrillator, lead parameters dysfunction, left ventricular assist device

\section{1 | INTRODUCTION}

Left ventricular assist device (LVAD) implantation has become an important therapeutic option in patients with end-stage heart failure, many of them being already implanted with an implantable cardioverter-defibrillator (ICD) at the time of LVAD surgery.

The concomitant use of an ICD and an LVAD has been shown to be safe but right ventricular (RV) ICD lead parameters dysfunction has been described after LVAD implantation. ${ }^{1-5}$ Indeed, previous studies have reported significant reductions in RV sensing amplitude or RV pacing impedance, sometimes requiring lead revisions. ${ }^{3,5}$

However, the impact of LVAD implantation on preexisting ICD leads and their resultant clinical implications have only been reported in limited single-center studies. Furthermore, data regarding LVAD interferences are mainly based on case reports and small series. The purpose of this large, multicenter observational study was to describe device interferences occurring in LVAD recipients and characterize the incidence of ICD lead parameters dysfunction after LVAD implantation.

\section{2 | METHODS}

\section{1 | Study design}

The ASSIST-ICD (Clinicaltrials.gov identifier: NCT02873169) is a retrospective, multicenter observational study of durable mechanical circulatory support devices implanted in 659 patients treated in 19 tertiary care centers in France. The study methods have been previously reported. ${ }^{6}$ Notably, it was initially conceived with the scope to address issues connected with decision-making in the arrhythmology and cardiac electrophysiology field among LVAD recipients (rate of the occurrence of ventricular arrhythmias under LVAD therapy and impact of such arrhythmia in LVAD recipients with/without implantable cardioverter defibrillator). Inclusion criterion was age $\geq 18$ at LVAD implantation; use of one among three continuous-flow LVADs (HeartMate II [Abbott Inc., Chicago, IL], HeartWare HVAD [Medtronic Inc., Minneapolis, MN], and Jarvik 2000 LVAD [Jarvik Heart Inc., NY]) as the primary implantation between February 2006 and December 2016 
was included. Exclusion criteria were total artificial heart placement or pulsatile flow LVAD, history of heart transplant, and receipt of a VentrAssist device (Ventracor, Chatswood, New South Wales, Australia). This study was approved by the regional ethic committees, the French Advisory Committee on the Treatment of Research Information in the Field of Health (CCTIRS), and the French National Commission of Informatics and Civil Liberties (CNIL). A nonopposition letter was sent to the patients, as requested by French authorities for retrospective studies.

\subsection{Study population and data collection}

Among the overall data collected, we were able to conduct a subanalysis focus on ICD lead parameters evolution between pre- and post-LVAD implantation. In this study, only the patients implanted with an ICD prior to LVAD associated with a complete ICD interrogation prior/after LVAD surgery were included in the analysis. Baseline data-including demographic characteristics, cardiac disease, and heart failure history, echocardiography-were collected from hospital files for all patients. Perioperative data (such as the necessity of a combined surgery) were also collected. The manufacturer was recorded for each ICD device and ICD ventricular lead (i.e., Abbott [Chicago, IL], Biotronik [Berlin, Germany], Boston Scientific [Marlborough, MA], Medtronic [Minneapolis, MN], and MicroPort [Shanghai, China]). ICD lead parameters, including RV lead sensing, lead pacing impedance, and RV pacing thresholds, were also collected using the last and first ICD interrogation reports available prior and after LVAD implantation, respectively.

\section{3 | ICD lead parameters dysfunction}

As previously proposed, ${ }^{7}$ significant ICD lead parameters dysfunction following LVAD implantation were defined as follows: (a) $>50 \%$ decrease in sensing threshold, (b) pacing lead impedance increase/decrease by $>100 \Omega$, and (c) $>50 \%$ increase in pacing threshold.

\section{4 | Endpoints}

The primary endpoint was the incidence of ICD lead parameters dysfunction following LVAD implantation. Secondary endpoint was the clinical impact of ICD lead parameters dysfunction in an LVADrecipients population.

\section{5 | Statistical analysis}

Qualitative variables are expressed as number (percentage) while continuous data are represented as mean \pm SD or median (interquartile range) depending on their distribution using the Kolmogorov-Smirnov test. Categorical variables were compared using the $\chi^{2}$ tests or Fisher's
TABLE 1 Baseline characteristics

\begin{tabular}{|ll|}
\hline Variables & Values \\
\hline Patients & 122 \\
\hline Age, years & $61.0(54.0-67.1)$ \\
\hline Men & $105(86.0)$ \\
\hline Body mass index, kg/mš & $25.4(23.5-27.8)$ \\
\hline Heart failure etiology & \\
\hline Ischemic & $64(52.5)$ \\
\hline Idiopathic & $47(38.5)$ \\
\hline Other & $11(9.0)$ \\
\hline Delay ICD implantation-LVAD implantation, months & $19.7(8.1-44.1)$ \\
\hline ICD in secondary prevention & $19(15.6)$ \\
\hline LVEF prior to LVAD, \% & $20.0(15.0-25.0)$ \\
\hline LVEDD prior to LVAD, mm & $72.0(67.7-79.0)$ \\
\hline CRT prior to LVAD & $65(53.3)$ \\
\hline LVAD & $13(10.7)$ \\
\hline HeartMate II & $7(5.7)$ \\
\hline HeartWare & $81(66.4)$ \\
\hline Jarvik2000 & $28(22.9)$ \\
\hline Combined surgery with LVAD & $160.1 m 09$ \\
\hline
\end{tabular}

Abbreviations: CRT, cardiac resynchronization therapy; ICD, implantable cardioverter-defibrillator; LVAD, left ventricular assist device; LVEDD, left ventricular end-diastolic diameter; LVEF, left ventricular ejection fraction.

exact test. Continuous variables were compared using Student's $t$ test or the Mann-Whitney $U$-test for two-group comparisons. A $P$-value $<.05$ was considered statistically significant. The analyses were performed with the SPSS statistical package, version 25.0 (SPSS Inc., Chicago, IL)

\section{3 | RESULTS}

\section{1 | Baseline characteristics}

Among the 659 LVAD recipients enrolled in the study, a total of 402 had an ICD prior to LVAD. Among the 402 patients, 91 died within the postoperative period (median time to death: 12.0 [4.0-31.0] days), and two were heart transplanted during the postoperative period. None of these 93 patients had ICD interrogation post-LVAD surgery. In this subset of 309 remaining patients, 63 patients had no device interrogation prior and after LVAD implantation, 35 and 37 had no device interrogation prior and after LVAD surgery, respectively, 15 were dependent from the RV pacing, and 37 had at least one missing data and were not included in the final analysis.

A total of 122 patients had a complete ICD interrogation report available prior and after LVAD surgery (i.e., sensing, pacing impedance, and pacing threshold). After LVAD implantation, patients were followed during a median period of 9.1 (2.5-22.1) months. Patients' characteristics are summarized in Table 1 . Briefly, patients were mostly men 
(A)

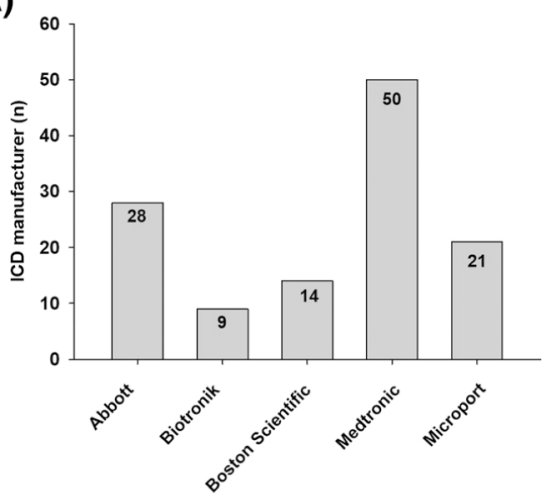

(B)

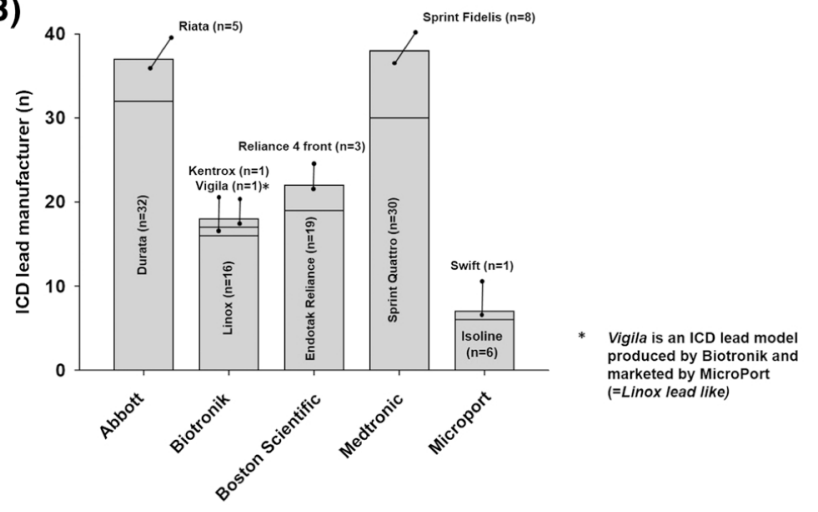

(C)
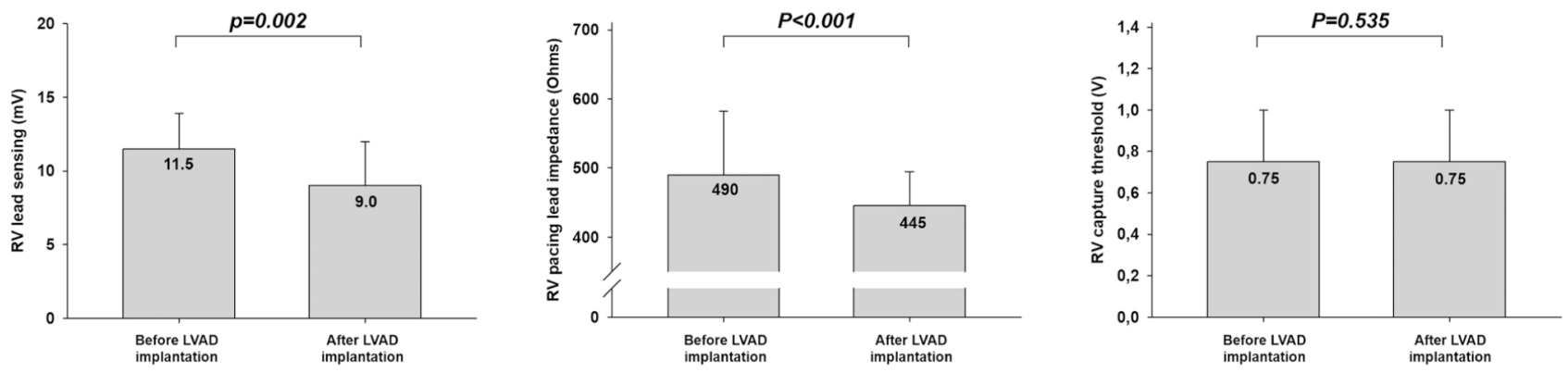

FI G URE 1 ICDs and leads manufacturer (panels A and B). Overall lead parameters change from prior to after LVAD implantation (panel C). Abbreviations: ICD, implantable cardioverter-defibrillator; LVAD, left ventricular assist device; RV, right ventricle

(86.0\%) with an ischemic cardiomyopathy (52.5\%). Nineteen (15.6\%) patients were implanted in secondary prevention and up to $50 \%$ received a cardiac resynchronization therapy - defibrillator (CRT-D) device. HeartMate II was implanted in $66.4 \%$ of the LVAD candidates, and $<10 \%$ had a cardiac surgery combined with LVAD implantation.

\subsection{Changes in ICD lead parameters after LVAD implantation}

Among the subset of 122 patients with a complete ICD interrogation report available prior and after LVAD surgery, ICD interrogation was performed during a median time of 48.0 (17.0-153.5) days prior and 52.0 (15.5-138.5) days after LVAD implantation. The distribution of ICDs and leads manufacturers is depicted in Figure 1 (panels $A$ and B). As illustrated in Figure 1 (panel C), the overall RV sensing and pacing impedance significantly decreased after LVAD implantation (i.e., from 11.5 [7.9-13.8] to 9.0 [6.3-12.0] $\mathrm{mV} ; P=.002$ and from 490.0 [430.0-582.0] to 445.5 [399.0-494.0] $\Omega, P<.001$, respectively), while the RV pacing threshold remained unchanged. Interestingly, these changes were only driven by some manufacturers: the RV sensing decrease being significant only for Boston Scientific ICD leads, whereas impedance reduction being significant for Abbott and Medtronic ICD leads (Figure 2). Of note, Biotronik and MicroPort leads did not present any significant changes.

A total of 67 (55\%) patients exhibited at least one significant lead dysfunction: 17 (15\%) patients exhibited a decrease by $>50 \%$ of RV sensing, 51 (42\%) had an increase/decrease by $>100 \Omega$ of RV pacing impedance, and 24 (20\%) experienced an increase by $>50 \%$ of RV pacing threshold (Figures 3-5, panel A). Main ICD lead models with parameters dysfunction are summarized in Figures 3-5 (panel B) but no statistical difference was noted between manufacturers depending on the number of altered parameters per lead (Figure 6). However, $\geq 50 \%$ of the ICD leads commercialized by Abbott, Biotronik, Boston Scientific, and Medtronic exhibited at least one significant parameter alteration after LVAD implantation. During follow-up, no patient required an ICD lead revision due to these dysfunctions.

Lastly, changes in ICD lead parameters were also analyzed depending on the sensing vector characteristic (i.e., true bipolar or integrated bipolar). Results are showed in Table 2. Among the 122 leads, a total of 92 and 30 leads were classified as true bipolar and integrated bipolar, respectively. Briefly, no difference was found in both groups but there is a trend toward higher RV sensing reduction in integrated bipolar lead between pre- and post-LVAD implantation. Additionally, a higher rate of true bipolar lead experienced $>50 \%$ increase in pacing threshold despite this result was insignificant.

\subsection{Clinical impact of lead dysfunction}

Among the 122 patients, a total of eight patients (7\%) experienced at least one inappropriate therapy during follow-up. Seven patients received a total of 18 inappropriate ICD shocks, and two received a total of three inappropriate anti-tachycardia therapies. Of note, atrial 

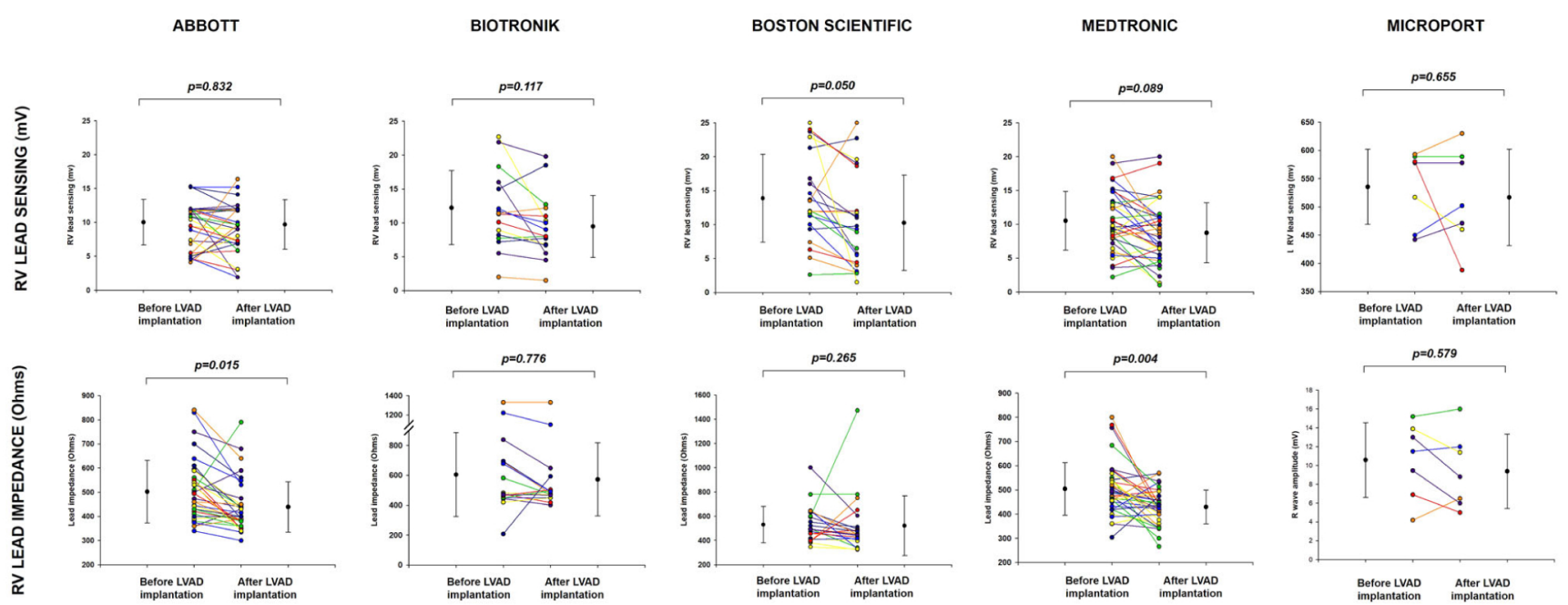

FIG URE 2 RV sensing and pacing impedance variation per manufacturer. Abbreviations: ICD, implantable cardioverter-defibrillator; RV, right ventricle [Color figure can be viewed at wileyonlinelibrary.com]

(A)

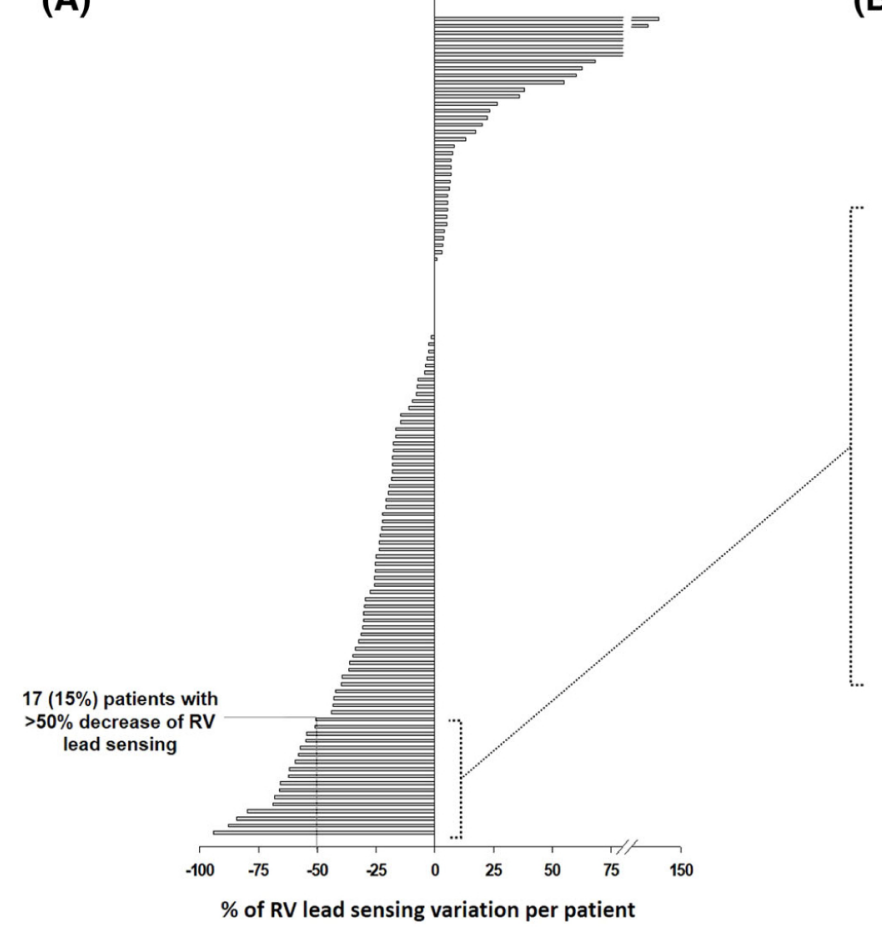

(B)

Leads with $>50 \%$ decrease of sensing

Leads without $>50 \%$ decrease of sensing

FIG URE 3 RV sensing variation per patient (panel A). ICD lead models with $>50 \%$ of RV sensing decrease (panel B). Abbreviations: ICD, implantable cardioverter-defibrillator; RV, right ventricle [Color figure can be viewed at wileyonlinelibrary.com]

TA B LE 2 ICD lead parameters variation depending on the true bipolar or integrated bipolar sensing vectors characteristics

\begin{tabular}{|c|c|c|c|}
\hline & True bipolar ICD lead $(n=92)$ & Integrated bipolar ICD lead $(n=30)$ & $P$ value \\
\hline RV sensing variation between pre-/post-LVAD (mV) & $-0.7(-3.1$ to +0.5$)$ & $-2.4(-4.8$ to +0.1$)$ & .079 \\
\hline RV pacing threshold variation between pre/post-LVAD (V) & $0.0(-0.2$ to +0.2$)$ & $0.0(-0.2$ to 0.0$)$ & .127 \\
\hline RV pacing impedance variation between pre/post-LVAD $(\Omega)$ & $-49.0(-131.5$ to 0.0$)$ & $-33.0(-67.0$ to +29.0$)$ & .286 \\
\hline$>50 \%$ decrease in RV sensing & $13(14.1)$ & $4(13.3)$ & .846 \\
\hline$>50 \%$ increase in pacing threshold & $22(23.9)$ & $2(6.7)$ & .072 \\
\hline RV pacing lead impedance increase/decrease by $>100 \Omega$ & $41(44.6)$ & $10(33.3)$ & .384 \\
\hline
\end{tabular}


(A)

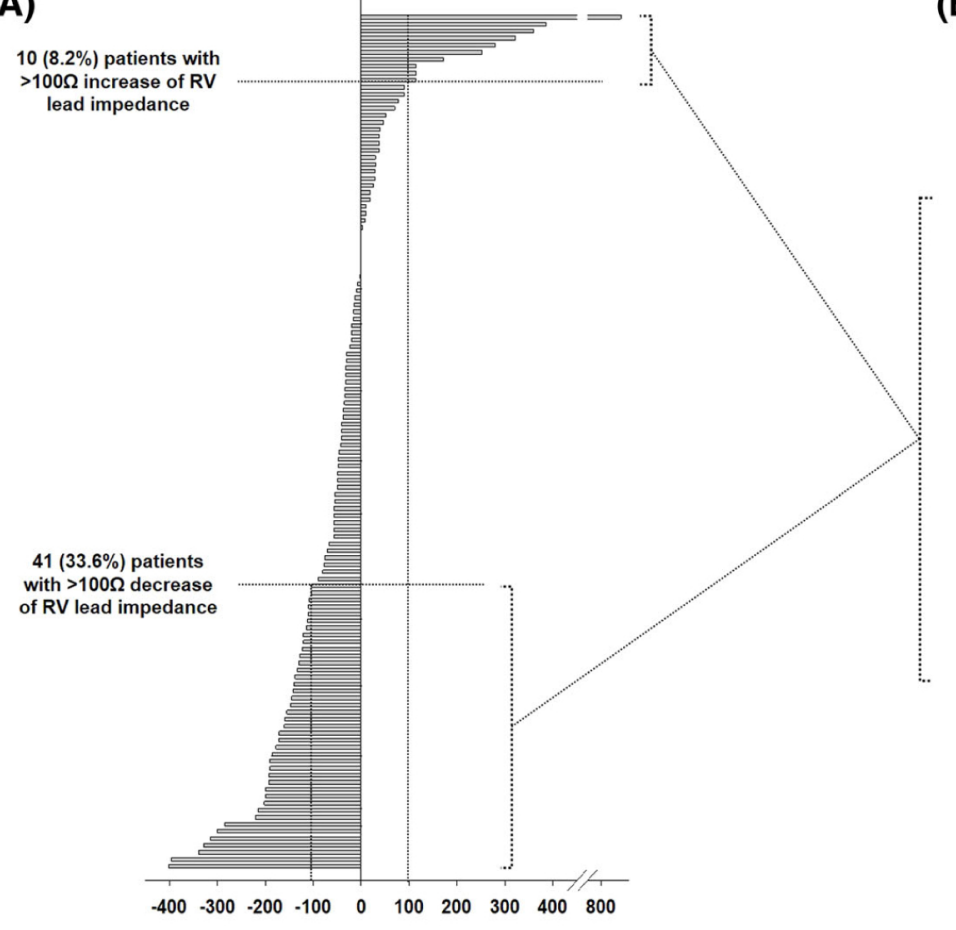

(B)

Leads with $>100 \Omega$ of impedance variation

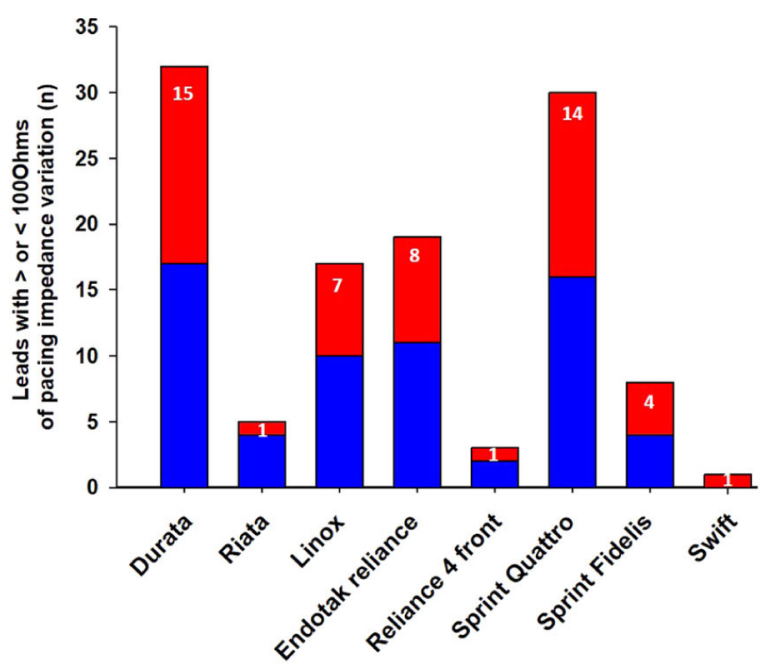

RV lead impedance variation per patient (Ohms)

FIGURE 4 RV impedance variation per patient (panel A). ICD lead models with an increase/a decrease by $>100 \Omega$ of pacing impedance (panel B). Abbreviations: ICD, implantable cardioverter-defibrillator; RV, right ventricle [Color figure can be viewed at wileyonlinelibrary.com]

(A)

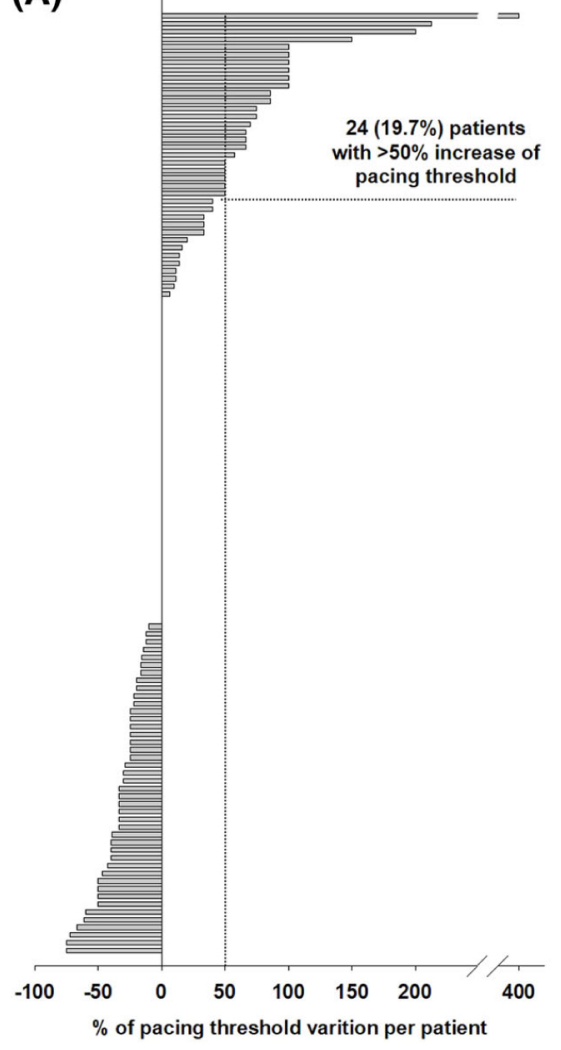

(B)
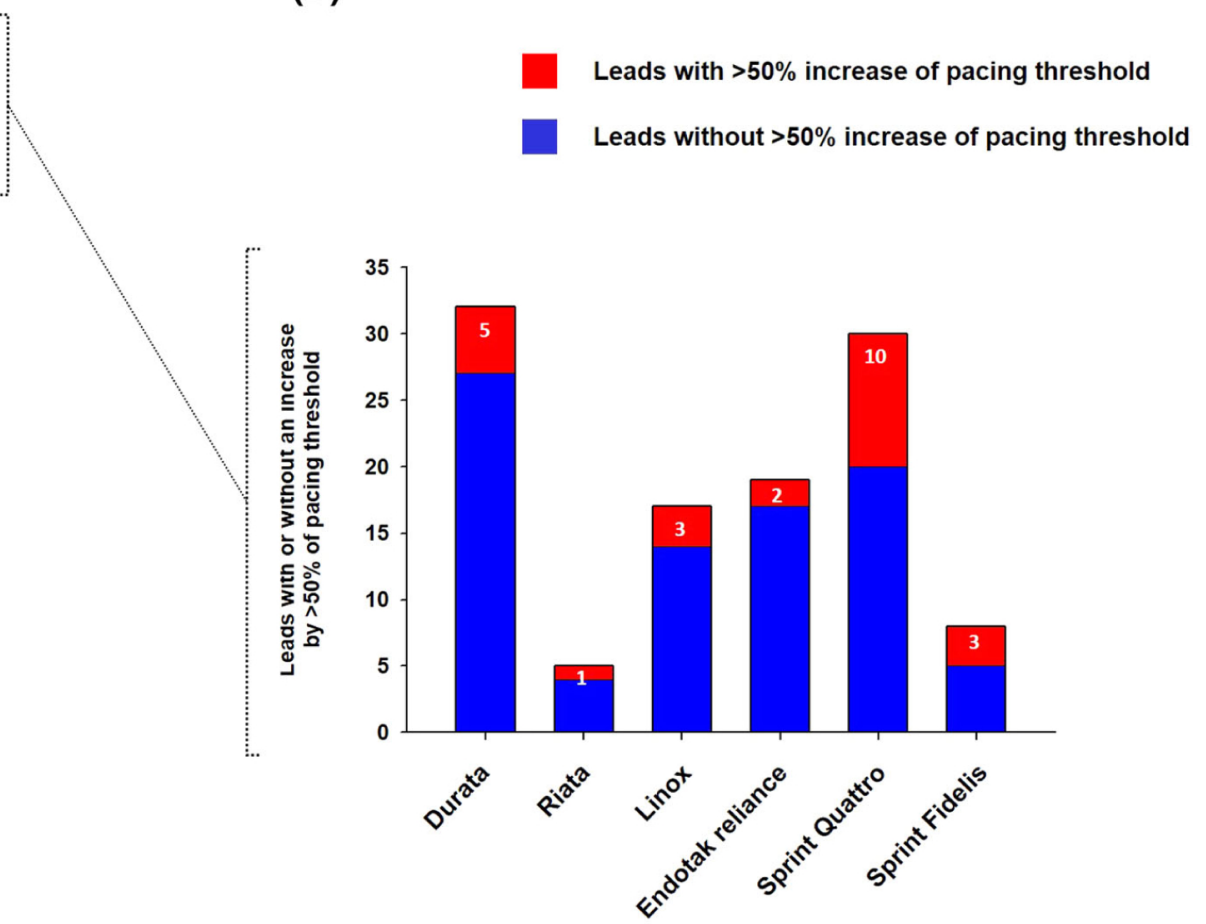

FIGURE 5 RV pacing threshold variation per patient (panel A). ICD lead models with > 50\% increase of pacing threshold (panel B). Abbreviations: ICD, implantable cardioverter-defibrillator; RV, right ventricle [Color figure can be viewed at wileyonlinelibrary.com] 


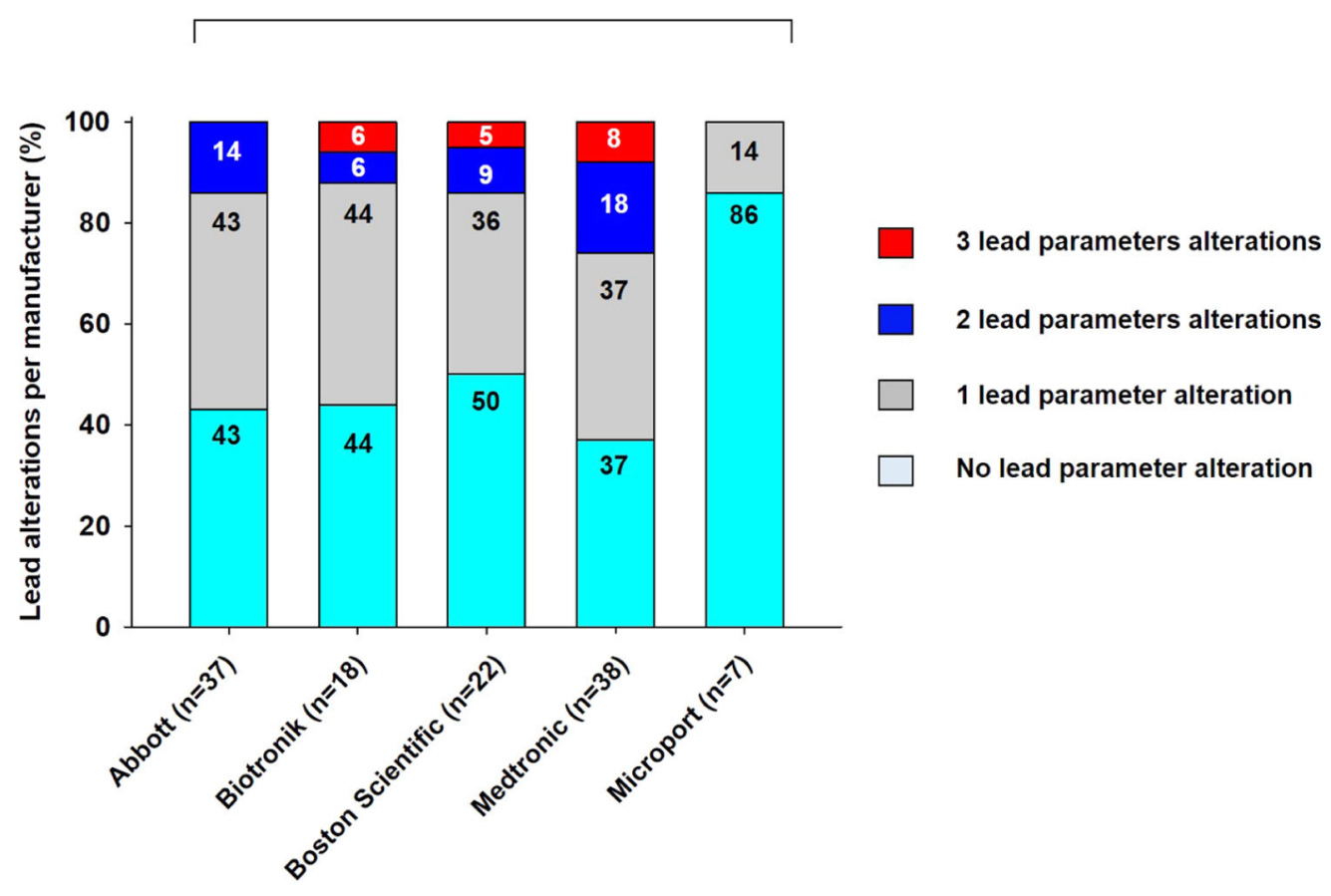

FIG URE 6 Rate of lead parameters alteration per manufacturer. Abbreviations: ICD, implantable cardioverter-defibrillator; RV, right ventricle [Color figure can be viewed at wileyonlinelibrary.com]

fibrillation, myopotential oversensing, and T wave oversensing were involved in six, one, and one patients experiencing inappropriate therapy, respectively. Notably, no inappropriate ICD therapy has been triggered by LVAD noise oversensing. Additionally, patients with $>50 \%$ decrease in RV sensing received a significant higher rate of inappropriate therapies compared to those without $>50 \%$ sensing reduction $(4 / 17,23.5 \%$ vs $4 / 105,1 \%, P=.012)$. Of note, patients with $>50 \%$ decrease in sensing had significantly more history of atrial fibrillation at baseline (15/17 vs 55/109; $P=.008)$.

Ventricular tachyarrhythmias occurred in 52 (43\%) patients with a total of 1151 VT and 79 VF episodes (i.e., 4.5 [2.0-20.0] ventricular arrhythmias episodes per patients). Among the 52 patients, all ventricular arrhythmia was successfully detected and treated by the device, and patients received a median number of 2.0 (1.0-10.0) antitachycardia pacing therapies and $1.0(0.0-3.0)$ ICD shock to restore sinus rhythm. Importantly, no episode of ventricular arrhythmia undersensing occurred during follow-up, even in those patients with RV sensing decrease.

\section{4 | DISCUSSION}

\section{1 | Main results}

Our study is the largest to accurately evaluate the impact of LVAD implantation on ICD lead function. The main results of this study are as follows: (a) significant lead parameters dysfunction is common after LVAD implantation and occurred in up to $50 \%$ of the patients; (b) all dysfunction could be managed conservatively by reprogramming, and no lead revision was required during follow-up; and (c) despite lead dysfunction, all ventricular arrhythmias episodes were successfully treated.

\subsection{ICD lead alteration following LVAD implantation}

Few studies including a limited number of patients have assessed the impact of LVAD implantation on preexisting ICD lead function. Indeed, in a cohort of 15 patients, Foo et al demonstrated a significant alteration of lead parameters (i.e., $R$ wave amplitude, RV pacing impedance, and RV pacing threshold) at 6 months after LVAD implantation. ${ }^{1}$ Similar results have been described in a population of 23 LVAD recipients with a significant decrease in $\mathrm{R}$ wave amplitude and $\mathrm{RV}$ impedance (from 12 to $9 \mathrm{mV}$ and from 520 to $456 \Omega$, respectively) after LVAD surgery. ${ }^{3}$ In a larger study enrolling 44 patients, authors have noted that lead characteristics (i.e., lead diameter and lead manufacturer) may influence lead function following LVAD implantation. ${ }^{4}$ Indeed, smaller lead diameters $(<2.7 \mathrm{~mm}$ ) and leads with pace/sense electrode surface areas $\leq 5.7 \mathrm{~mm}^{2}$ were associated with a significant decrease in sensing amplitude. Additionally, Medtronic ICD leads exhibited significant decrease in sensing threshold compared to Abbott and Boston Scientific leads. Our study confirms these data among a larger population of 122 patients. Indeed, we found that after a median postoperative period of 52 days, the overall LVAD population had a significant decrease in RV sensing and pacing impedance. However, based on 
ICD lead brand, only Boston Scientific leads had a significant decrease in RV sensing, and Abbott/Medtronic leads had a significant decrease in RV pacing impedance compared to the preoperative period. Importantly, we also found that $55 \%$ of LVAD recipients presented at least one significant alteration of lead parameters (i.e. $>50 \%$ RV sensing decrease $=15 \%$ of the patients, $>100 \Omega$ of pacing impedance variation $=41 \%$ of the patients, and $>50 \%$ pacing threshold increase $=20 \%$ of the patients). These results are supported by a recent study showing in a cohort of 32 patients that RV sensing decreased by $>50 \%$ in $22 \%$ of the patients. However, the impact of LVAD implantation on RV pacing threshold was more modest with only $6 \%$ of the patients experiencing an increase by $>50 \%$ of pacing threshold. ${ }^{5}$ Importantly, we also showed that $\geq 50 \%$ of ICD leads commercialized by Abbott, Biotronik, Boston Scientific, and Medtronic, exhibited at least one significant parameter alteration after LVAD implantation, suggesting that the manufacturer lead design does not seem to influence these dysfunctions.

The reasons explaining the alterations of ICD lead parameters after LVAD implantation remain unclear. Some authors have hypothesized that the manipulation of the heart during surgery may result in a micro dislodgment of the ICD lead. ${ }^{2,4}$ Additionally, an elegant study has suggested that LV unloading associated with a concomitant leftward shifting of the septum could directly modify the myocardium conformation near to the lead and alter lead parameters. Indeed, in a population of 32 patients, authors have monitored the lead function at different step of LVAD implantation and observed an important variation of RV sensing during surgery. ${ }^{1}$ Lastly, myocardial ischemia and edema secondary to LVAD surgery could also possibly contribute to temporary dysfunction of lead properties. However, in our study, lead testing was performed during a median time of 52 days after surgery, suggesting that the potential deleterious effect of the LVAD on ICD leads is not a transient postoperative phenomenon. Indeed, a recent review has suggested that lead parameters changes persisting beyond the 30 days postoperatively were mostly definitive. ${ }^{7}$

\section{3 | Clinical implication}

Despite the fact that up to $50 \%$ of the patients experienced significant lead dysfunction, such changes were not associated with a deleterious clinical impact. Indeed, no lead replacement was necessary, and all ventricular arrhythmias were appropriately detected and treated by the ICD. Importantly, no undersensing of ventricular arrhythmias occurred. Additionally, inappropriate therapies episodes were mostly related to atrial fibrillation, and lead dysfunctions were not involved in such cases. In the light of these data, ICD with altered lead parameters appears to be relatively safe in LVAD recipients, and the need for lead revisions should be carefully discussed in this population. Indeed, recent data demonstrate that ICD-related procedure after pump implantation is an independent predictor of LVAD infection. ${ }^{8}$ In patients with significant lead dysfunction, a conservative management should be preferred, especially if ICD reprogramming is sufficient to overcome such dysfunction allowing a proper device functioning.

\section{5 | LIMITATIONS}

This study has some limitations, including its retrospective design that may have affected the results. Importantly, a relatively low number of ICD recipients had complete ICD interrogation data and were included in the final analysis. Additionally, the high-voltage lead impedance was not recorded, and consequently we are not able to provide a complete evaluation of lead parameters alteration after LVAD implantation. However, previous study did not report major high voltage-impedance variation after LVAD implantation (defined as an increase or decrease by $>10 \Omega[\mathrm{U}]$ after LVAD surgery). ${ }^{8}$ Furthermore, our measurements were performed after a median period of 52 days following LVAD surgery, and we did not report long-term ICD interrogation to observe any parameters improvement. Nevertheless, long-term lead parameters improvement is unusual beyond the 30 days postoperative period.

\section{6 | CONCLUSION}

ICD lead dysfunctions are common after LVAD implantation. Patients with an ICD experience a significant decrease in RV sensing and RV pacing impedance after LVAD surgery. Additionally, more than 50\% of the LVAD-recipients experience at least one significant parameter alteration. However, these variations are not clinically relevant and lead revision is usually not required.

\section{ACKNOWLEDGMENTS}

This research was supported in part by the French Federation of Cardiology.

\section{REFERENCES}

1. Foo D, Walker BD, Kuchar DL, et al. Left ventricular mechanical assist devices and cardiac device interactions: an observational case series. Pacing Clin Electrophysiol. 2009;32:879-887.

2. Ambardekar AV, Lowery CM, Allen LA, et al. Effect of left ventricular assist device placement on preexisting implantable cardioverterdefibrillator leads. J Card Fail. 2010;16:327-331.

3. Boudghène-Stambouli $F$, Boulé $S$, Goéminne $C$, et al. Clinical implications of left ventricular assist device implantation in patients with an implantable cardioverter-defibrillator. J Interv Card Electrophysiol. 2014;39:177-184

4. Thomas IC, Cork DP, Levy A, et al. ICD lead parameters, performance, and adverse events following continuous-flow LVAD implantation. Pacing Clin Electrophysiol. 2014;37:464-472.

5. Hu YL, Kasirajan V, Tang DG, et al. Prospective evaluation of implantable cardioverter-defibrillator lead function during and after left ventricular assist device implantation. JACC Clin Electrophysiol. 2016;2:343-354.

6. Galand V, Flécher E, Auffret V, et al. Predictors and clinical impact of late ventricular arrhythmias in patients with continuous-flow left ventricular assist devices. JACC Clin Electrophysiol. 2018;4:1166-1175. 
7. Ho G, Braun OÖ, Adler ED, Feld GK, Pretorius VG, BirgersdotterGreen U. Management of arrhythmias and cardiac implantable electronic devices in patients with left ventricular assist devices. JACC Clin Electrophysiol. 2018;4:847-859.

8. Tattevin P, Flécher E, Auffret $\mathrm{V}$, et al. Risk factors and prognostic impact of left ventricular assist device-associated infections. Am Heart J. 2019;214:69-76. 